\section{Musculoskeletal Disorders-1}

\section{B.1 THE IMPACT OF MANUAL PATIENT HANDLING ON WORK ABILITY: A CROSS-SECTIONAL STUDY}

${ }^{1}$ Adriano Dias*, ${ }^{1}$ João Marcos Bernardes. ${ }^{1}$ Unesp - São Paulo State University, Botucatu Medical School, Botucatu, Brazil

\subsection{6/OEM-2019-EPI.91}

This cross-sectional study investigated the level of work ability (WA) and its association with manual patient handling $(\mathrm{MPH})$ in healthcare workers (HCW). Participants were HCWs working at the University Hospital of Botucatu Medical School, who were willing to participate and provided written informed consent. Of the 488 HCWs who were invited, 320 participated for a response rate of $65.6 \%$. Sixteen participants $(5 \%)$ were subsequently excluded from data analysis due to missing data. Data were collected using a self-administered anonymous paper questionnaire containing seven parts: manual patient handling, low back pain, demographic characteristics, occupational variables, psychosocial work conditions, lifestyle variables and work ability. Work ability was measured by the Brazilian version of the Work Ability Index (WAI). The association between WA and LBP was analyzed using a Poisson regression model. Initially, simple Poisson regression models with robust variance were conducted for each covariate. Those variables with a $\mathrm{p}$ value $\leq$ 0.25 were selected for inclusion in a multiple Poisson regression model with robust variance, and those with $\mathrm{p}<0.05$ were considered statistically significant. Most respondents were female $(83.6 \%)$, the mean age was 40.35 years (SD 9.74, range 22-66) and the average duration of employment was 10.67 years (SD 9.36, range 1-40). The WAI mean score was 38.03 points (SD 6.15, 95\% CI 37.33-38.72), and the prevalence of adequate WA (equal to or above 37 points) was $56.58 \%$. In the multiple Poisson regression model, MPH (PR 1.375, 95\% CI 1.038-1.821) was significantly associated with WA, even when adjusted for the covariates. Thus, hospital managers should plan and implement interventions to maintain healthcare workers' work ability, and these interventions should include the limitation of manual patient handling activities.

\section{O4B.2 PATTERNS AND PREDICTORS OF RETURN TO WORK AFTER MAJOR TRAUMA: A PROSPECTIVE, POPULATION BASED REGISTRY STUDY}

\footnotetext{
${ }^{1}$ Alex Collie*, ${ }^{1}$ Pamela Simpson, ${ }^{1}$ Peter Cameron, ${ }^{2}$ Shanthi Ameratunga, ${ }^{1}$ Jennie Ponsford, ${ }^{3}$ Ronan Lyons, 'Sandra Braaf, ${ }^{4}$ Andrew Nunn, ${ }^{5}$ James Harrison, ${ }^{1,3}$ Belinda Gabbe. 'Monash University, Melbourne, Australia; 'University of Auckland, Auckland, New Zealand; ${ }^{3}$ Swansea University, Swansea, UK; ${ }^{4}$ Austin Health, Heidelberg, Australia; ${ }^{5}$ Flinders University, Adelaide, Australia
}

\subsection{6/OEM-2019-EPI.92}

Background Employment is an important marker of functional recovery from injury. There are few population-based studies of long-term employment outcomes, and limited data on the patterns of return to work post injury.

Objectives This study sought to characterise patterns of engagement in work over the four-year period following major traumatic injury, and to identify factors associated with those patterns.
Method We conducted a population-based, prospective cohort study using the Victorian State Trauma Registry. A total of 1086 working age individuals, in paid employment or fulltime education before injury, were followed-up through telephone interview at $6,12,24,36$, and 48 months post-injury. Responses to return to work (RTW) questions were used to define four discrete patterns: early and sustained; delayed; failed; no RTW. Predictors of RTW patterns were assessed using multivariate multinomial logistic regression.

Results Slightly more than half of respondents (51.6\%) recorded early sustained RTW. A further 15.5\% had delayed and $13.3 \%$ failed RTW. One in five $(19.7 \%)$ did not RTW. Compared with early sustained RTW, predictors of delayed and no RTW included being in a manual occupation and injury in a motor vehicle accident. Older age and receiving compensation predicted both failed and no RTW patterns. Pre-injury disability was an additional predictor of failed RTW. Presence of co-morbidity was an additional predictor of no RTW.

Conclusions A range of personal, occupational, injury, health and compensation system factors influence RTW patterns after serious injury. Early identification of people at risk for delayed, failed or no RTW is needed so that targeted interventions can be delivered.

\section{B.3 THE IMPACT OF INCOME SUPPORT SYSTEMS ON HEALTHCARE QUALITY AND FUNCTIONAL CAPACITY IN WORKERS WITH LBP: A REALIST REVIEW}

Michael Di Donato*, Ross lles, Tyler Lane, Alex Collie. Monash University, Melbourne, Australia

\subsection{6/OEM-2019-EPI.93}

Background Low back pain (LBP) is a leading cause of work disability. While absent from work, workers with LBP may receive income support from a system such as workers' compensation or social security. Current evidence suggests that income support systems can influence recovery from LBP, but provides little evidence as to why and how these effects occur. This study examines how and in what contexts income support systems impact the healthcare quality and functional capacity of people with work disability and LBP.

Methods We performed a realist review, a type of literature review that seeks to explain how social interventions and phenomena in certain contexts generate outcomes, rather than simply whether or not they do. Five initial theories about the mechanisms of the relationship were developed, tested, and refined by acquiring and synthesising academic literature from purposive and iterative electronic database searching. This process was supplemented by grey literature searching for policy documents and legislative summaries, and semi-structured interviews with experts in income support, healthcare, and LBP.

Results Income support systems influence healthcare quality through healthcare funding restrictions, healthcare provider administrative burden, and allowing an employer to select healthcare providers. Income support systems influence worker functional capacity through the level of participation and share of income support funding required of employers, and through certain administrative procedures. These mechanisms are often exclusively context-dependent, and generate differing 